ИЗВЕСТИЯ АКАДЕМИИ НАУК ЭСТОНСКОИ ССР. ФИЗИКА * МАТЕМАТИКА

PROCEEDINGS OF THE ACADEMY OF SCIENCES OF THE ESTONIAN SSR. PHYSICS * MATHEMATICS

$1985,34,3$

\title{
К ТЕОРИИ ДВУХСТУПЕНЧАТОГО ВОЗБУЖДЕНИЯ ЛЮМИНЕСЦЕНЦИИ
}

\author{
(Представил В. Хиюняков)
}

1. Двухступенчатое возбуждение люминесценции двумя последовательными короткими пикосекундными импульсами позволяет изучать сверхбыструю релаксацию в молекулах $\left[{ }^{1-3}\right]$. Наглядная (и несколько упрощенная) картина заключается в следующем. Первый импульс переводит часть молекул из начального 0 в первое возбужденное электронное состояние 1. Задержанный на временной интервал $T$ второй импульс переводит часть молекул из 1 в следующее возбужденное электронное состояние 2. Экспериментально измеряется интенсивность всего вторичного свечения $2 \rightarrow 0$ (основной вклад в которое вносит обычная люминесценция) как функция от времени задержки $T$ между импульсами, и отсюда определяется время жизни в возбужденном электронном состоянии 1.

Цель данной работы - теоретическое описание двухступенчатого возбуждения люминесценции в рамках теории вторичного свечения примесного центра (см. $\left[{ }^{4,5}\right]$, а также $\left.\left[{ }^{6}\right]\right)$.

2. Принимая за начало отсчета времени момент прохождения центра максимумом первого возбуждающего импульса, интенсивность вторичного свечения $2 \rightarrow 0 I(T, t)$ в момент времени $t$ можно записать следующим образом:

$$
I(T, t) \sim \frac{d}{d t}\left(W_{0}(T, t)-W(T, t)\right)
$$

Здесь $W(T, t)$ - вероятность найти систему в момент времени $t$ в состоянии $2, W_{0}(T, t)$ - та же вероятность, но в том случае, когда состояние 2 энергетически не затухает $*, T-$ интервал времени между максимумами возбуждающих импульсов.

В экспериментах $\left[{ }^{1}\right]$ измерялась суммарная по времени интенсивность вторичного свечения

$$
I(T) \equiv \int_{-\infty}^{\infty} d t I(T, t) \sim W_{0}(T, \infty)
$$

(в (2) предполагается, что $\left.W(T,-\infty)=W_{0}(T,-\infty)=W(T, \infty)=0\right)$. Она пропорциональна числу молекул, возбужденных в состояние 2 двумя импульсами, в предположении, что в состоянии 2 нет процессов тушения.

* Учет фазовой релаксации в состоянии 2 не существен, поскольку рассматривается суммарная по времени интенсивность $I(T)$. 
3. Вероятность $W_{0}(T, \infty)$ возбуждения состояния 2 двумя следующими друг за другом с интервалом $T$. одинаковыми импульсами описывается формулой второго порядка теории возмущения

$$
\begin{gathered}
I(T) \sim W_{0}(T, \infty)=\iint_{-\infty}^{\infty} d t_{1} d t^{\prime}{ }_{1} S\left(t_{1}-T, t_{1}^{\prime}-T\right) \times \\
\times \int_{-\infty}^{t_{1}} d t_{2} \int_{-\infty}^{t_{1}^{\prime}} d t^{\prime}{ }_{2} S\left(t_{2}, t_{2}^{\prime}\right) a\left(t^{\prime}{ }_{1}-t_{1}, t^{\prime}{ }_{1}-t^{\prime}{ }_{2}, \quad\left(t_{1}-t_{2}\right),\right.
\end{gathered}
$$

где $S\left(x_{1}, x_{2}\right)$ - корреляционная функция импульса,

$$
\begin{aligned}
& a\left(t^{\prime}{ }_{1}-t_{1}, t^{\prime}{ }_{1}-t^{\prime}{ }_{2}, t_{1}-t_{2}\right)=\left\langle v_{1 \omega}^{+} \exp \left(i\left(H_{c}+i \gamma_{c}\right)\left(t_{1}-t_{2}\right)\right) \times\right. \\
& \times v_{2 \omega}^{+} \exp \left(i H_{c}\left(t^{\prime}{ }_{1}-t_{1}\right)\right) v_{2 \omega} \exp \left(-i\left(H_{c}-i \gamma_{c}\right)\left(t^{\prime}{ }_{1}-t^{\prime}{ }_{2}\right)\right) \times \\
& \left.\times v_{1 \omega} \exp \left(-i H_{c}\left(t^{\prime}{ }_{2}-t_{2}\right)\right)\right\rangle_{c}
\end{aligned}
$$

- корреляционная функция трехуровневой системы. Здесь

$$
\begin{aligned}
& v_{1 \omega}=\langle\omega|\langle 0|V| \omega\rangle| \omega\rangle, \\
& v_{2 \omega}=\langle 0|\langle 0|V| 0\rangle| \omega\rangle
\end{aligned}
$$

- матричные элементы, описывающие уничтожение фотона частоты $\omega$, $V$ - гамильтониан взаимодействия, $H_{c}$ и $\gamma_{c}$ - гамильтониан и оператор затухания трехуровневой системы, 〈... $\rangle_{c}-$ знак усреднения по ансамблю колебаний.

4. Выберем корреляционную функцию импульсов в следующем виде:

$$
S\left(x_{1}, x_{2}\right)=\left\{\begin{array}{l}
2 \Delta \exp \left(i_{\omega}\left(x_{1}-x_{2}\right)-\Delta\left(x_{1}+x_{2}\right)-\delta\left|x_{1}-x_{2}\right|\right) ; \\
\text { если } x_{1}, x_{2}>0, \\
0 \text { в других случаях. }
\end{array}\right.
$$

Здесь $\omega$ - частота максимума импульса, $(2 \Delta)^{-1}-$ длительность импульса во времени, $2(\Delta+\delta)$ - спектральная ширина импульса.

Используем приближение Кондона. Релаксационные процессы в состоянии 1 будем описывать константами энергетической (продольной) $\gamma$ и фазовой (поперечной) $\Gamma$ релаксаций. Для описания эффектов поперечной релаксации в этом состоянии можно использовать результаты $\left[{ }^{4}\right]$. В результате корреляционная функция трехуровневой системы оказывается равной

$$
\begin{aligned}
& a\left(t^{\prime}{ }_{1}-t_{1}, t^{\prime}{ }_{1}-t^{\prime}{ }_{2}, t_{1}-t_{2}\right)=C \exp \left[i \Omega_{1}\left(t^{\prime}{ }_{2}-t_{2}\right)+\right. \\
& +i \Omega_{2}\left(t^{\prime}{ }_{1}-t_{1}\right)-\gamma\left(t_{1}-t_{2}+t^{\prime}{ }_{1}-t^{\prime}{ }_{2}\right)-\Gamma\left(\left|t^{\prime}{ }_{1}-t_{1}\right|+\right. \\
& \left.+t^{\prime}{ }_{1}-t^{\prime}{ }_{2}+t_{1}-t_{2}+\left|t^{\prime}{ }_{2}-t_{2}\right|-\left|t^{\prime}{ }_{2}-t_{1}\right|-\left|t^{\prime}{ }_{1}-t_{2}\right|\right) .
\end{aligned}
$$

Здесь $\Omega_{1}, \Omega_{2}$ - частоты электронных переходов мсжду нсчальным 0 и промежуточным 1 , и между промежуточным 1 и конечным 2 уровнями, C - константа.

5. После подстановки формул (5), (6) в (3) и интегрирования получим для интенсивности вторичного свечения $I(T)$ следующую формулу

$$
\begin{aligned}
I(T)= & 4 \Delta^{2} C\left\{\alpha _ { 1 } \left[C_{1} \exp (-2 \Delta T) / 4 \Delta+C_{2} \exp (-2 \gamma T) / 2(\Delta+\gamma)+\right.\right. \\
+ & \left(C_{3} \exp \left(-i\left(\Omega_{1}-\omega\right) T\right)+C_{4} \exp \left(i\left(\Omega_{1}-\omega\right) T\right)\right) \times \\
& \times \exp (-(\Delta+\delta+\gamma+\Gamma) T)]+\alpha_{2} C_{5}[\exp (-2 \Delta T) / 4 \Delta+ \\
& \left.\left.+g_{4} \exp \left(i\left(\Omega_{1}-\omega\right) T-(\Delta+\delta+\gamma+\Gamma) T\right)\right]+C . C .\right\},
\end{aligned}
$$

где 


$$
\begin{aligned}
& \alpha_{1}=\left[i\left(\Omega_{2}-\omega\right)-(\Delta+\delta+\gamma+\Gamma)\right]^{-1}, \\
& \alpha_{2}=\left[i\left(\Omega_{1}+\Omega_{2}-2 \omega\right)-2(\Delta+\delta)\right]^{-1}, \\
& g_{1}=\left[i\left(\Omega_{1}-\omega\right)+\Delta+\delta-\gamma+\Gamma\right]^{-1} \\
& g_{2}=\left[i\left(\Omega_{1}-\omega\right)-\Delta+\delta+\gamma+\Gamma\right]^{-1}, \\
& g_{3}=\left[i\left(\Omega_{1}-\omega\right)-\Delta-\delta+\gamma+\Gamma\right]^{-1}, \\
& g_{4}=\left[i\left(\Omega_{1}-\omega\right)-(3 \Delta+\delta+\gamma+\Gamma)\right]^{-1}, \\
& C_{1}=g_{2}^{*}\left[g_{3}-g_{2}((\delta+\Gamma) /(\gamma-\Delta)+1)\right], \\
& C_{2}=\left|g_{2}\right|^{2}[(\delta+\Gamma) /(\gamma-\Delta)-1], \\
& C_{3}=g_{1} g_{2} g_{4}^{*}, \quad C_{4}=g_{2}^{*} g_{4}\left(g_{1}^{*}+g_{3}\right), \\
& C_{5}=-g_{2}^{*} g_{3} .
\end{aligned}
$$

6. Из формулы (7) видно, что, с одной стороны, выражение для интенсивности вторичного свечения $I(T)$ можно разделить на две части:

$$
I(T)=I_{1}(T)+I_{2}(T)
$$

где $I_{1}(T)$ учитывает слагаемые, пропорциональные $\alpha_{1}$, и соответствует возбуждению состояния 2 двухступенчатыми однофотонными переходами $0 \stackrel{\oplus}{\rightarrow} 1 \stackrel{\oplus}{\rightarrow} 2$, а $I_{2}(T)$ учитывает слагаемые, пропорциональные $\alpha_{2}$, и соответствует возбуждению одноступенчатым двухфотонным переходом $0 \stackrel{2 \omega}{\rightarrow} 2$ (т. е. состояние 1 реально не возбуждается). Но это деление, вообще говоря, формальное, что проявляетсл в возможности появления отрицательных значений одного из компонентов (в рассматриваемых нами примерах появились отрицательные значения $\left.I_{2}(T)\right)$. Всегда положительна только суммарная вероятность $I(T)$.

С другой стороны, $I(T)$ содержит три различным образом затухающих компонента:

$$
\begin{gathered}
I_{\mathrm{I}}(T) \sim \exp (-2 \Delta T), \quad I_{\mathrm{II}}(T) \sim \exp (-2 \gamma T), \\
I_{\mathrm{III}}(T) \sim \cos \left(\left(\Omega_{1}-\omega\right) T\right) \exp (-(\Delta+\delta+\gamma+\Gamma) T) .
\end{gathered}
$$

Затухание $I_{I}(T)$ определяется длительностью возбуждающих импульсов, затухание $I_{\mathrm{II}}(T)$ - скоростью продольной релаксации состояния 1 . Член $I_{\mathrm{II}}(T)$ осциллирует; его затухание определяется как параметрами возбуждающих импульсов, так и параметрами ( $\gamma$ и Г) состояния 1 , т. е. $I_{\mathrm{III}}(T)$ описывает интерференцию между $I_{\mathrm{I}}(T)$ и $I_{\mathrm{II}}(T)$. Таким образом, по кинетике затухания $I(T)$ можно просто определить параметры $\gamma$ и $\Delta$. Время поперечной релаксации $\Gamma^{-1}$ прямо по зависимости $I$ от $T$ определить нельзя.

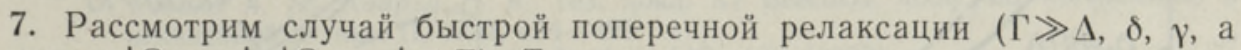
также $\left.\left|\Omega_{1}-\omega\right|,\left|\Omega_{2}-\omega\right| \ll \Gamma\right)$. Тогда

$$
\begin{gathered}
I(T) \sim 2 \Delta C \Gamma^{-2}\{\exp (-2 \Delta T) /(\gamma-\Delta)-2 \Delta \exp (-2 \gamma T) / \\
\left./\left(\gamma^{2}-\Delta^{2}\right)+2(\Delta+\delta) \exp (-2 \Delta T) /\left[\left(\Omega_{1}+\Omega_{2}-2 \omega\right)^{2}+4(\Delta+\delta)^{2}\right]\right\}
\end{gathered}
$$

т. е. интенсивность вторичного свечения $I(T)$ пропорциональна квадрату времени поперечной релаксации $I(T) \sim \Gamma^{-2}$.

8. Для анализа формул (7), (8) были проведены расчеты на ЭВМ. При расчете интенсивности вторичного свечения $I(T)$ для разных расстроек от резонанса перехода $0 \rightarrow 1$ (рис. 1) выяснилссь, что спектры в случаях 
$\Omega_{1}=\omega+x$ и $\Omega_{1}=\omega-x$ различны. При увеличении расстройки сначала максимум сдвигается в область $T>0$, а затем (при достаточно больших $\left.\left|\Omega_{1}-\omega\right|\right)$ зависимость от $T$ становится осциллирующей.
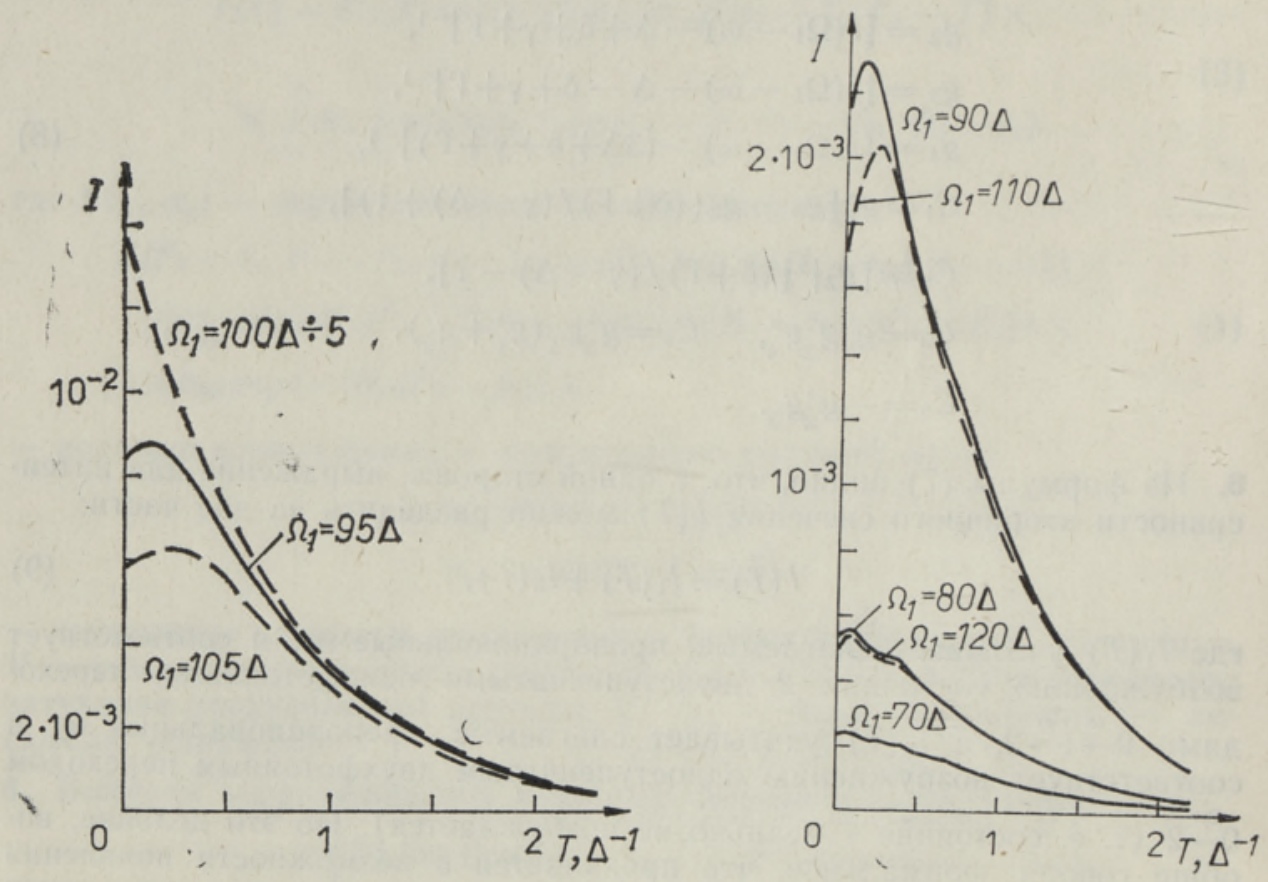

Рис. 1. Интенсивность вторичного свечения $I(T)$ для разных расстроек от резонанса перехода $0 \rightarrow 1$. Параметры: $\gamma=\Delta, \Gamma=2 \Delta, \delta=0,5 \Delta, \omega=100 \Delta, \Omega_{2}=102 \Delta$.

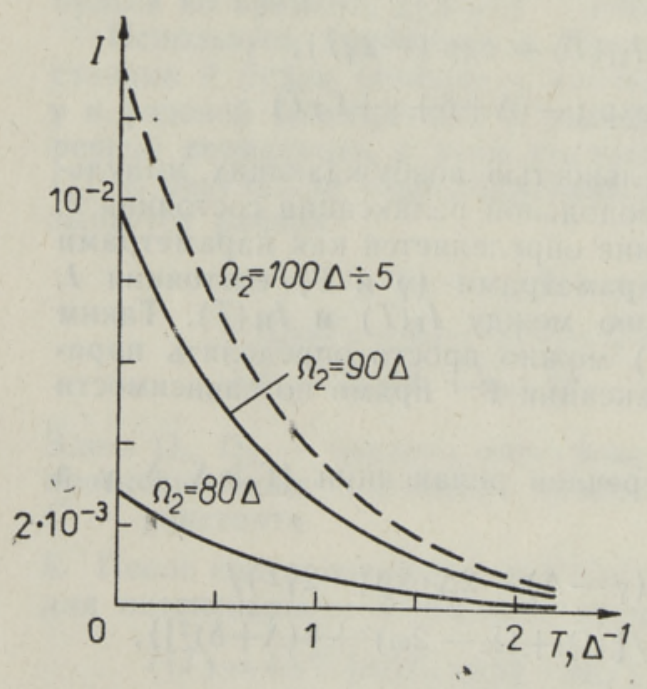

Рис. 2. Интенсивность вторичного свечения $I(T)$ для разных расстроек от резонанса перехода $l \rightarrow 2$. Параметры: $\gamma=\Delta$, $\Gamma=2 \Delta, \quad \delta=0,5 \Delta, \quad \omega=100 \Delta, \Omega_{1}=101 \Delta$.

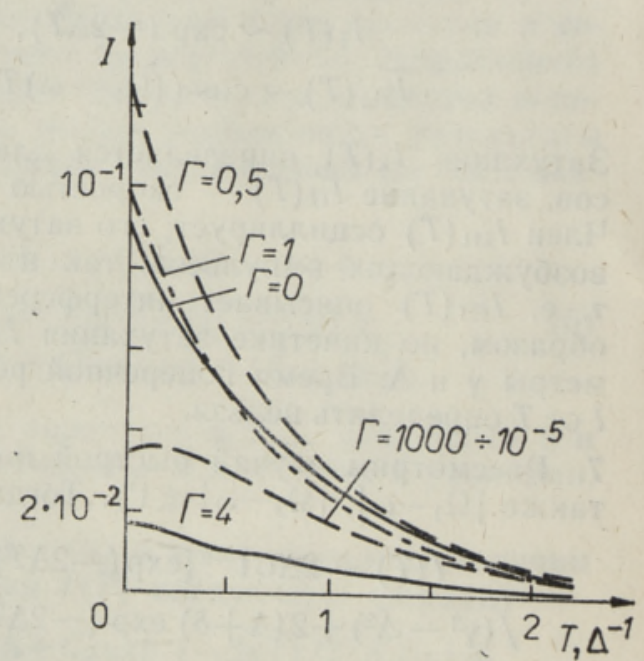

Pис. 3. Интенсивность вторичного свечения $I(T)$ для разных значений константы поперечной релаксации Г. Параметры: $\gamma=\Delta, \delta=0,5 \Delta, \omega=100 \Delta, \Omega_{1}=101 \Delta$, $\Omega_{2}=102 \Delta$ 
Рис. 4. Интенсивность вторичного свечения $I(T)$, рассчитанная с использованием параметров экспериментальной работы [ $\left.{ }^{1}\right]$ : длительность импульсов 5 пс, спектральная ширина импульсов $0,4 \pi^{-1}$ (т. е. $\Delta=\delta=$ $\left.=0,1 \mathrm{nc}^{-1}\right)$, время затухания возбужденного электронного состояния 1 $(2 \gamma)^{-1}=26$ пс (кривая 1) и $\sim \exp (-T / 26)$ (кривая 2).

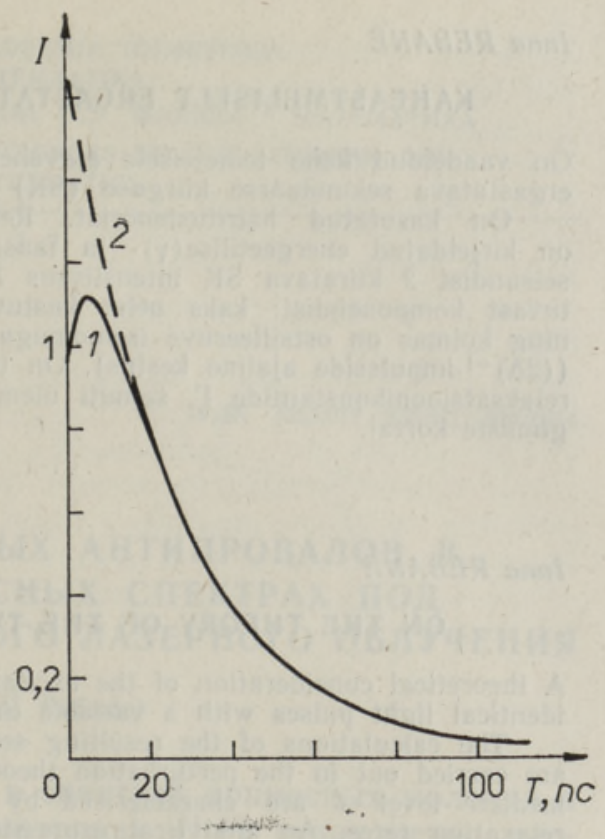

Зависимость интенсивности $I(T)$ от расстройки резонанса переходов $0 \rightarrow 1$ и $1 \rightarrow 2$ (рис. 2) разная. Нет зависимости от знака $\Omega_{2}-\omega$.

Имеется нетривиальная зависимость интенсивности $I(T)$ от константы поперечной релаксации Г (рис. 3).

При анализе интенсивности вторичного свечения $I(T)$, рассчитанной с использованием параметров экспериментальной работы [ $\left.{ }^{1}\right]$ (рис. 4), выяснилось, что $I(T)$ имеет максимальное значение при временной задержке $T=5$ пс. При этой задержке между импульсами возбуждение вторичного свечения $I(T)$ наиболее эффективное. Это происходит потому, что именно через 5 пс после прохождения максимума первого импульса электроннос состояние 1 возбуждено максимально: первый !Імпульс практически прошел (импульс затухает по закону $\sim \exp (-t / 5)$, а затухание возбужденного электронного состояния 1 еще не успело существенно сказаться на его заселенности (состояние 1 затухает по закону $\sim \exp (-t / 26))$. Поэтому вероятность перехода $1 \rightarrow 2$ при задержке $T \sim 5$ пс наибольшая.

Автор благодарен В. Хижнякову, К. К. Ребане и А. Ребане за обсуждение работы, А. Туулу за выполнение расчетов на ЭВМ.

\section{ЛИТЕРАТУРА}

1. Каарли Р., Ребане А. Изв. АН ЭССР. Физ. Матем., 30, № 3, 287--289 (1981); Ребане А. К., Каарли Р. К. Тез. докл. ХІ Всесоюз, конф. по когерентной и нелинейной оптике. II, 1982, 494-495.

2. Rentzepis, P. M. Chem. Phys. Lett., 2, № 1, 117-121 (1968).

3. Ippen, E. P., Shank, C. V., Woerner, R. L. Chem. Phvs. Lett., 46, № 1, 20-23 (1977).

4. Hizhnyakov, V., Tehver, I. Phys. status solidi, 21, № 2, 755-768 (1967).

5. Hizhnyakov, V., Tehver, I. Phys. status solidi (b) 39, № 1, 67-78 (1970); 82, K89-K93 (1977).

6. Хижняков В.АВ., Ребане И. К. Ж. эксперим. и теор. физ., 74, вып. 3, 885-896 (1978).

\section{Ннститут физики \\ Академии наук Эстонской ССР}

Поступила в редакцию $7 /$ VI 1984 


\section{KAHEASTMELISELT ERGASTATAVA LUMINESTSENTSI TEOORIAST}

On vaadeldud kahe teineteisele ajavahemiku $T$ pärast järgneva ühesuguse impulsiga ergastatava sekundaarse kiirguse (SK) protsessi kolmenivoolises süsteemis $\{0,1,2\}$. On kasutatud häiritusteooriat. Relaksatsiooniprotsesse vahepealses seisundis 1 on kirjeldatud energeetilise $(\gamma)$ - ja faasi $(\Gamma)$-relaksatsioonikonstandiga. On leitud lõppseisundist 2 kiiratava SK intensiivsus $I(T)$, mis koosneb kolmest erineval viisil kustuvast komponendist: kaks neist kustuvad vastavalt $\sim \exp (-2 \gamma T)$ ja $\sim \exp (-2 \Delta T)$ ning kolmas on ostsilleeruva iseloomuga, kirjeldades kahe esimese vahelist interferentsi $\left((2 \Delta)^{-1}\right.$-impulsside ajaline kestus). On tehtud SK $I(T)$ masinarvutused erinevate faasirelaksatsioonikonstantide $\Gamma$, samuti üleminekute $0 \rightarrow 1$ ja $1 \rightarrow 2$ erinevate resonantstingimuste korral.

\section{Inna REBANE}

\section{ON THE THEORY OF THE TWO-STEP EXCITED FLUORESCENCE}

A theoretical consideration of the excitation of a three-level system $\{0,1,2\}$ with two identical light pulses with a variable time delay $T$ between them, is presented.

The calculations of the resulting secondary emission (SE) from the upper level 2 are carried out in the perturbation theory approximation. The relaxations on the intermediate level 1 are characterized by phenomenological energy $(\gamma)$ and phase $(\Gamma)$ relaxation rates. An analytical expression is calculated for the level 2 SE intensity $I(T)$ dependence on delay $T . I(T)$ is shown to contain two exponential decay terms $\sim \exp (-2 \gamma T)$ and $\sim \exp (-2 \Delta T)$, and an exponential decay $\sim \exp (-(\Delta+\delta+\gamma+\Gamma) T)$ oscillatory term describing the interference between the two components $\left((2 \Delta)^{-1}\right.$ is the duration and $2(\Delta+\delta)$ is the spectral width of excitation pulses). The results of the computer calculations of $I(T)$ are presented for various $\Gamma$ values, and also for different $0 \rightarrow 1$ and $1 \rightarrow 2$ transition resonance conditions. 